- Case Report

\title{
A Brief Psychotic Episode with Depressive Symptoms in Silent Right Frontal Lobe Infarct
}

\author{
Salziyan Badrin ${ }^{1, *}$, Noraini Mohamad², Nor Akma Yunus', Maryam Mohd Zulkifli' \\ 'Department of Family Medicine, School of Medical Sciences, Health Campus, Universiti Sains Malaysia, Kelantan, Malaysia \\ ${ }^{2}$ School of Dental Sciences, Health Campus, Universiti Sains Malaysia, Kelantan, Malaysia
}

Psychiatric symptoms may be related to a silent cerebral infarct, a phenomenon that has been described previously in literature. Acute psychosis or other neuropsychiatric symptoms including depression may present in stroke patients and patients with lesions either within the prefrontal or occipital cortices, or in subcortical areas such as the basal ganglia, thalamus, mid-brain, and brainstem. Psychosis in clinical stroke or in silent cerebral infarction is uncommon and not well documented in the literature. Neurological deficits are the most common presentation in stroke, and nearly a third of patients that suffer a stroke may experience psychological disorders such as depression and anxiety, related to physical disability. The present case report describes an elderly female patient who presented with hallucinations and depressive symptoms, and was discovered to have a recent right frontal brain infarction, without other significant neurological deficits.

Keywords: Depression; Psychotic Disorders; Stroke; Stroke Manifestations; Aged 


\section{INTRODUCTION}

Stroke may be associated with psychiatric symptoms such as depression, anxiety, and apathy. ${ }^{1)}$ A silent cerebral infarction occurs without clinical symptoms, but can be detected on imaging modalities. ${ }^{2)} \mathrm{De}-$ pression in elderly people has been reported to be associated with silent cerebral infarcts. ${ }^{2)}$ Depression is commonly related to disability and impairment in interpersonal functioning that can occur after a stroke event. The data in literature about psychosis in stroke is limited. An earlier study has indicated that acute psychosis or neuropsychiatric symptoms may be present in stroke patients, and in patients with lesions either within the prefrontal or occipital cortices, or in subcortical areas such as the basal ganglia, thalamus, mid-brain, and brainstem. ${ }^{3)}$ A few studies have reported psychosis as the initial presentation of

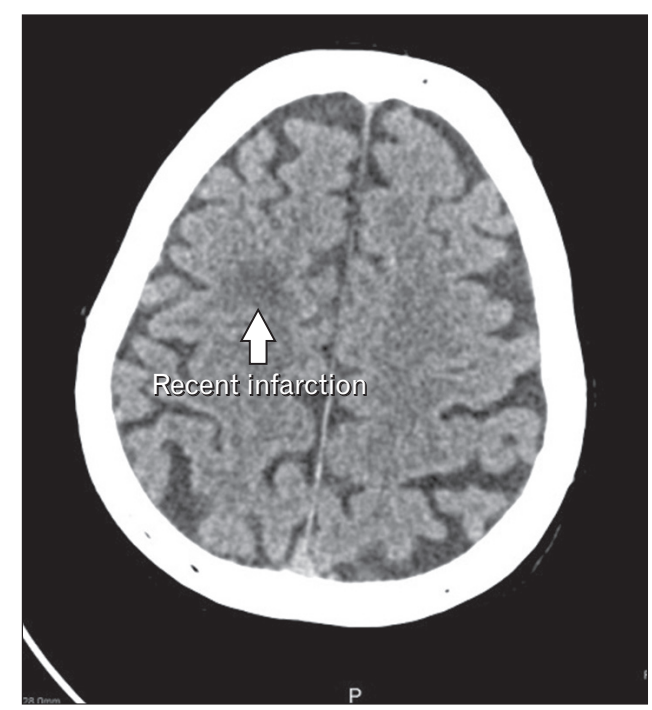

Figure 1. Computed tomography scan of the brain showing the recent infarction at the right frontal region (arrow). stroke. ${ }^{2,4)}$

\section{CASE REPORT}

A 73-year-old woman with underlying diabetes and hypertension, but without a psychiatric history, complained of insomnia associated with visual and auditory hallucinations prior to sleeping, all of which persisted for one month. She described the hallucinations as hearing a voice talking about 'killing' each time she tried to sleep, and she reported one episode of seeing a black shadow. She had other depressive symptoms such as anhedonia, easily fatigue, decrease in energy, poor appetite, and low mood, all of which appeared for one month. The patient did not report suicidal thoughts or ideation, nor had she attempted suicide. There was no history of aggressive behavior or talking to oneself. She did not have any memory complaints, and there was no significant neurological deficit or history of fall or trauma. The patient denied having been treated for stroke in the past, which was confirmed by the family members. She was able to perform daily living activities such as dressing, eating, going to the toilet, taking a shower, and walking unassisted. She was able to handle her own finances but there were some limitations in performing activities such as travelling, using the phone, shopping, and doing laundry, hence, she relied on help from her children.

A physical examination showed that she was alert and conscious with good orientation. Her blood pressure was 154/78 mm Hg, her pulse was 84 beats per minute with regular rhythm and good volume, and her capillary blood sugar level was $6.5 \mathrm{mmol} / \mathrm{L}$. Her speech was clear with no slurring; however, the tone was low. There was no abnormal movement seen, the patient demonstrated good insight, and her elderly cognitive assessment questions score was 9 out of 10 . A mini mental state examination was not done at the initial visit. Neurological examination revealed normal muscle tone, power with the absence of ankle jerk reflexes bilaterally, and other sensory findings consistent with peripheral neuropathy. Electrocardiograms showed the presence
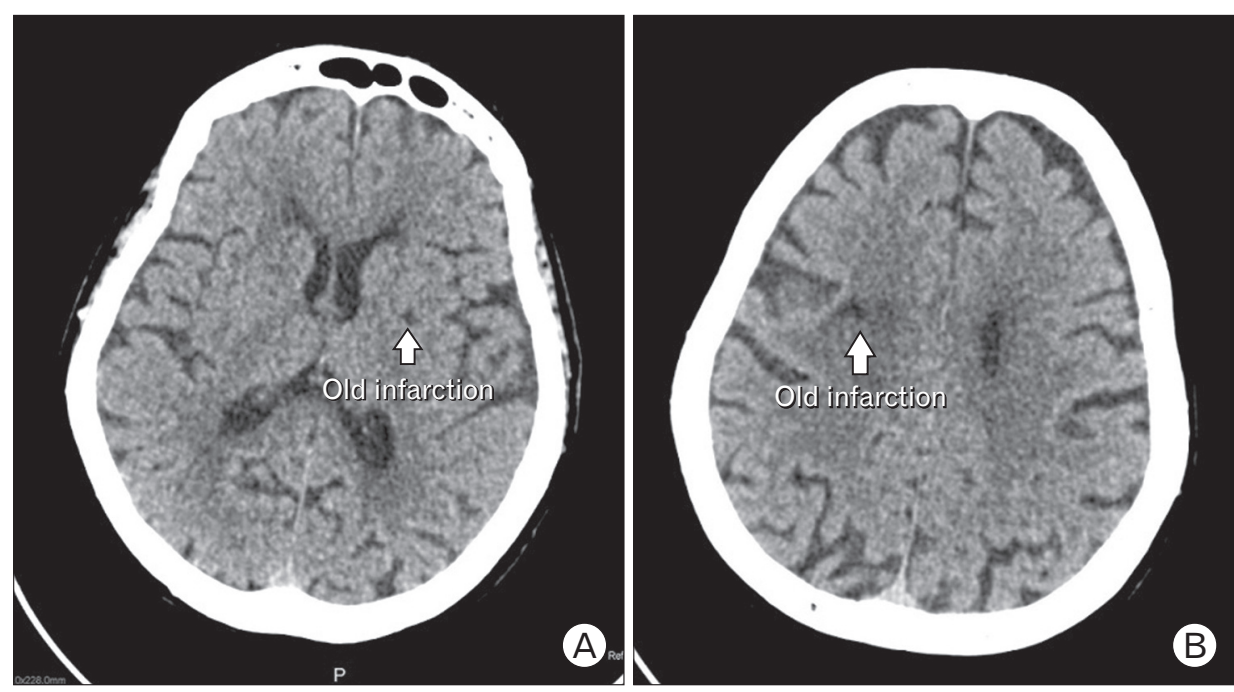

Figure 2. (A, B) Computed tomography scans of the brain showing multiple, well-defined hypodensities at the right centrum semiovale and the left lentiform nucleus (old infarction, arrow). 
of left ventricular hypertrophy without acute ischemic changes. A computed tomography (CT) scan of the brain with contrast was arranged and revealed the presence of a recent cerebral infarct in the right frontal area (Figure 1) with multiple old cerebral infarcts (well-defined hypodensities at the right centrum semiovale, right corona radiate, right thalamus, anterior and posterior limb of the right interior capsule, genu of the left internal capsule, bilateral lentiform nucleus, and both external capsules) (Figure 2A, B). Other tests such as those for thyroid function, renal function, and lipid profile were within acceptable ranges.

The diagnosis of depression with psychotic symptoms was made based on the clinical assessment, and the patient was prescribed a low dose of risperidone twice daily (initial $0.5 \mathrm{mg}$ twice daily) for a short duration, together with a selective serotonin reuptake inhibitor (SSRI) antidepressant, fluvoxamine, with a dose of $50 \mathrm{mg}$ once daily. A short course of benzodiazepine (alprazolam $0.5 \mathrm{mg}$ ) was administered asneeded to help her to sleep. Aspirin $150 \mathrm{mg}$ was prescribed daily for the unnoticed stroke, whereas oral hypoglycemic and antihypertensive agents were optimized to achieve the targeted control. Subsequent visits revealed improvement in her symptoms and condition.

\section{DISCUSSION}

Brain imaging studies, like CT and other modalities, are important for detecting possible secondary causes of neuropsychiatric illness, especially in elderly patients. A CT brain scan with contrast in this case accidentally revealed a recent infarct in the right frontal lobe, and multiple old infarct lesions. Based on the CT findings, it is difficult to conclude whether the current clinical symptoms were due to the recent infarct or due to an old infarct. A recent meta-analysis showed that the prevalence of depression after a stroke is $29 \%$. Depression can occur within two years of a stroke event, with a peak prevalence between approximately three to six months after a stroke, and a decrease in prevalence after two years of the stroke. ${ }^{1,5,6)}$ Post-stroke depression is the result of disruption in the neural circuits involved in mood regulation; this may be caused by ischemic brain lesions occurring concomitantly with psychosocial risk factors, such as a history of major depression, neurotic personality type, or social isolation. ${ }^{6,7)}$ The presence of other co-morbid illnesses may also contribute to post-stroke depression. ${ }^{6,7)}$ Certain sites of ischemic lesions such as the left anterior, left basal ganglia, and the area near the frontal pole are reported to be important lesion sites for post-stroke depression, but the facts about lesion sites as a risk factor for post-stroke depression are still debatable.

Psychosis is rarely reported to be a manifestation of cerebral infarction and is not common in stroke patients. A study performed by Fujikawa et al. ${ }^{2)}$ suggested that psychiatric symptoms may present as a manifestation of silent cerebral infarction. Psychotic symptoms occur in stroke may vary, and these may depend on the location of infarct lesions within the brain. ${ }^{3)}$ Frontal lobe dysfunction causing impairment in reality-check pathways is theorized to produce delusion, hallucinations, and dysregulation of inhibitory control of the ponto-geniculateoccipital system, and may contribute to the development of visual hal- lucinations. ${ }^{3)}$ The exact mechanism of psychosis in stroke remains unclear. Treatment with antipsychotic medications may provide symptomatic relief. ${ }^{3)}$

The presence of major or minor depression may negatively affect the patient's ability to perform daily activities like going to work, and interpersonal functioning. The presence of depression as early as one month after stroke onset may correlate with mortality within 1-2 years. ${ }^{8)}$ Hence, if it is left untreated, depression may worsen the degree of disability and increase the risk of mortality. There are studies that report favorable effects of SSRIs such as fluoxetine and escitalopram, which decrease depression-related disability, depressive and anxious symptoms, and neurological deficits in patients with stroke. ${ }^{1,9)}$ The benefits of SSRIs in patients without depression are also observed in a small sample and limited trials; however, the results are still inconclusive. Larger sample sizes and well-designed trials are still needed to determine whether SSRIs should be routinely given to patients after stroke. ${ }^{1)}$

In conclusion, acute onset of neuropsychiatric symptoms in elderly individuals without a history of previous psychiatric disorders should prompt a thorough workup to assess the causes of psychiatric symptoms, and comprehensive treatment should then be administered to the affected patients.

\section{CONFLICT OF INTEREST}

No potential conflict of interest relevant to this article was reported.

\section{REFERENCES}

1. Pedroso VS, Souza LC, Brunoni AR, Teixeira AL. Post stroke depression: clinics, etiopathogenesis and therapeutics. Arch Clin Psychiatry (Sao Paulo) 2015;42:18-24.

2. Fujikawa T, Yamawaki S, Touhouda Y. Incidence of silent cerebral infarction in patients with major depression. Stroke 1993;24:1631-4.

3. McMurtray A, Tseng B, Diaz N, Chung J, Mehta B, Saito E. Acute psychosis associated with subcortical stroke: comparison between basal ganglia and mid-brain lesions. Case Rep Neurol Med 2014;2014:428425.

4. Santos S, Alberti O, Corbalan T, Cortina MT. Stroke-psychosis: description of two cases. Actas Esp Psiquiatr 2009;37:240-2.

5. Srivastava A, Taly AB, Gupta A, Murali T. Post-stroke depression: prevalence and relationship with disability in chronic stroke survivors. Ann Indian Acad Neurol 2010;13:123-7.

6. Whyte EM, Mulsant BH. Post stroke depression: epidemiology, pathophysiology, and biological treatment. Biol Psychiatry 2002;52:253-64.

7. Dieguez S, Staub F, Bruggimann L, Bogousslavsky J. Is poststroke depression a vascular depression? J Neurol Sci 2004;226:53-8.

8. Nishiyama Y, Komaba Y, Ueda M, Nagayama H, Amemiya S, Katayama Y. Early depressive symptoms after ischemic stroke are associated with a left lenticulocapsular area lesion. J Stroke Cerebrovasc Dis 2010;19:184-9.

9. Wiart L, Petit H, Joseph PA, Mazaux JM, Barat M. Fluoxetine in early poststroke depression: a double-blind placebo-controlled study. Stroke 2000;31:1829-32. 